\title{
Visual cues combined with treadmill training to improve gait performance in Parkinson's disease: a pilot randomized controlled trial
}

Clinical Rehabilitation 2016, Vol. 30(5) 463-47I (c) The Author(s) 2015 Reprints and permissions: sagepub.co.uk/journalsPermissions.nav DOI: $10.1177 / 0269215515588836$ cre.sagepub.com

@SAGE

\author{
Cornelia Schlickk',2, Alina Ernst', Kai Bötzel ${ }^{3}$, \\ Annika Plate', Olena Pelykh' and Josef Ilmberger'
}

\begin{abstract}
Objective: To evaluate the effects of visual cues combined with treadmill training on gait performance in patients with Parkinson's disease and to compare the strategy with pure treadmill training.

Design: Pilot, exploratory, non-blinded, randomized controlled trial.

Setting: University Hospital of Munich, Germany.

Subjects: Twenty-three outpatients with Parkinson's disease (Hoehn and Yahr stage II-IV).

Interventions: Patients received 12 training sessions within five weeks of either visual cues combined with treadmill training $(n=I 2)$ or pure treadmill training $(n=I I)$.

Main measures: Outcome measures were gait speed, stride length and cadence recorded on the treadmill. Functional tests included the Timed Up and Go Test, the Unified Parkinson's Disease Rating Scale and the Freezing of gait-questionnaire. Assessments were conducted at baseline, after the training period and at two months follow-up.

Results: After the training period $(n=20)$, gait speed and stride length had increased in both groups $(p \leqslant 0.05)$. Patients receiving the combined training scored better in the Timed Up and Go Test compared with the patients receiving pure treadmill training $(p \leqslant 0.05)$. At two months follow-up $(n=13)$, patients who underwent the combined training sustained better results in gait speed and stride length $(p \leqslant 0.05)$ and sustained the improvement in the Timed Up and Go Test $(p \leqslant 0.05)$.

Conclusions: This pilot study suggests that visual cues combined with treadmill training have more beneficial effects on gait than pure treadmill training in patients with a moderate stage of Parkinson's disease. A large-scale study with longer follow-up is required.
\end{abstract}

\section{Keywords}

Parkinson's disease, gait, rehabilitation, cueing, treadmill training

Received: 3I March 20I4; accepted: 4 May 2015

'Department of Orthopedic Surgery, Physical Medicine and Rehabilitation, University Hospital of Munich (LMU), Munich, Germany

${ }^{2}$ German Center for Vertigo and Balance Disorders, LudwigMaximilians-University, Munich, Germany

${ }^{3}$ Department of Neurology, University Hospital of Munich (LMU), Munich, Germany

\footnotetext{
Corresponding author:

Cornelia Schlick, Department of Orthopedic Surgery, Physical Medicine and Rehabilitation, University Hospital of Munich (LMU), Marchioninistrasse I5, D-8I 377 Munich, Germany. Email: cornelia.schlick@med.uni-muenchen.de
} 


\section{Introduction}

Gait disturbances are among the most disabling motor symptoms of Parkinson's disease, leading to a substantial decline in mobility and independence, high rates of falls and related injuries, and a reduction in quality of life. ${ }^{1-4}$ Although pharmacological interventions are an essential part of therapy and surgical treatment has become a successful option, patients suffer from increasing mobility impairments as the disease progresses, ${ }^{5}$ indicating a need for alternative gait rehabilitation approaches.

Previous work has shown that cueing techniques can improve gait performance in patients with Parkinson's disease. ${ }^{5-9}$ Visual cues provided as markers on the walking surface, for example, seem to have a normalizing effect on the spatiotemporal gait parameters, specifically stride length. ${ }^{6}$

In a broader sense of the cueing concept, treadmill training has also proven to be effective in the treatment of Parkinsonian gait disorders. ${ }^{10-14}$ Frenkel-Toledo and colleagues suggest that the rotation of the treadmill belt operates as an external cue to generate a more rhythmic gait pattern. ${ }^{10}$

Utilizing the evidence supporting visual cueing and treadmill training, our research group developed an intervention strategy combining treadmill walking and visual cues. A case study showed that this method helped to substantially improve the walking ability of a patient with a severe stage of Parkinson's disease. ${ }^{15}$

The aim of the present study is to evaluate the effect of visual cueing in combination with treadmill training on walking performance, comparing this to pure treadmill training in a group of patients with a moderate to severe stage of the disease. We hypothesized that receiving visual cues during treadmill training is more successful in improving gait than receiving pure treadmill training.

\section{Method}

The study was performed at the Department of Orthopedic Surgery, Physical Medicine and Rehabilitation, University Hospital of Munich, Germany. Patients were recruited from medical records in our hospital and from local Parkinson associations to be assessed for eligibility. Inclusion criteria were: a diagnosis of idiopathic Parkinson's disease; disease severity of II to IV on the Hoehn and Yahr Scale; ${ }^{16}$ ability to stand independently and to walk on a treadmill (with body weight support, if required); sufficient visual capacity to see the cues. Exclusion criteria were: any other neurologic or orthopedic disorder affecting gait and postural stability; change in medication for the treatment of Parkinson's disease during the study period; cognitive impairment indicated by less than 24 points in the Mini-Mental State Examination (MMSE);17 severe cardiovascular disorders; vestibular dysfunctions.

The study was approved by the local Ethics committee and all patients were asked to provide written informed consent before participation.

Participants were allocated into two treatment groups by an independent person. Blocked randomization was used to ensure balanced group sizes. The randomization was conducted using numbered envelopes containing ten lots. Each of the envelopes was filled with five lots specifying "control" and another five specifying "training". Three envelopes were used. Patients could not be blinded to group allocation.

All patients were asked to regularly take their Parkinson medication. Testing and training procedures were individually planned and consecutively conducted at the same time of day during the "on"phase of their medication cycle.

\section{Training procedure}

Patients in both treatment groups completed 12 training sessions within five weeks (2-3 sessions each week) and exercised on a motorized medical treadmill $(\mathrm{h} / \mathrm{p} / \mathrm{cosmos}$ sports and medical GmbH, Nussdorf-Traunstein, Germany). The treadmill was equipped by a pressure-sensitive platform (zebris medical GmbH, Isny, Germany) under the rotating belt. This enabled the recording of the patient's foot pressure profile, his/her step length and cadence, as well as other gait parameters.

An instructed physiotherapist supervised the training sessions. During the first session, all patients trained for 20 minutes using their preferred walking speed (determined during the initial 
measurements at baseline, described below). Throughout the following sessions, training duration and treadmill speed were adapted to the patient's progress in activity level. All patients had reached a training duration of at least 35 to at most 45 minutes at the final session.

In Group 1, the training consisted of treadmill walking (with up to $20 \%$ of body weight support, if required) in combination with visual cues that were projected onto the treadmill belt. We used the custom-tailored RehaWalk ${ }^{\circledR}$ software (zebris medical $\mathrm{GmbH}$, Isny, Germany) for the projection of the cues. Before the training period started, the pressure profiles, i.e. the left and the right footprint of each patient, were recorded. The shapes of the subject's footprints were used as individual cues (Figure 1, available online). A projector (NEC Display Solutions Europe GmbH, Munich, Germany) at the front side of the treadmill displayed these in synchrony with the treadmill speed. Patients were instructed to step as precisely as possible onto the footprints during treadmill walking. The initial step length of each patient was taken to adjust the sagittal distance of the projected footprints. For the first training session, the sagittal distance of the projected cues was enlarged by $10 \%$ in comparison to the measured step length in order to facilitate improvement. For the following training sessions, the sagittal distance was continuously increased according to the individual progress in gait performance.

In Group 2, the training consisted of pure treadmill training (with up to $20 \%$ of body weight support, if required). No instructions on gait performance were given.

\section{Testing procedure}

All measurements were conducted at baseline (pre), after the training period (post) and after two months (follow-up) by two trained examiners who were not blinded to group allocation. The cognitive status was only assessed at baseline using the MMSE.

Gait speed, stride length and cadence were recorded over a period of 30 seconds during treadmill walking using the zebris gait analysis system. Severely affected patients (Hoehn and Yahr stage IV) were bodyweight supported up to $20 \%$. All other patients were secured during treadmill walking by the same system, but without body weight support. Before the initial measurement, patients were familiarized with treadmill walking for six minutes. During this adaption phase, the patients were instructed to identify their subjective preferred and maximum walking speed. Maximum walking speed was taken for the gait analysis.

Functional walking performance was assessed using the Timed Up and Go Test. ${ }^{18}$ If applicable, patients used their walking device for completing the test. To evaluate freezing of gait, the Freezing of Gait-Questionnaire was administered. ${ }^{19}$ Furthermore, the Motor section of the Unified Parkinson's Disease Rating Scale (UPDRS III) was used to evaluate overall motor abilities. ${ }^{20}$

\section{Data analysis}

Within a previous case study, one severely affected patient with Parkinson's disease achieved an improvement of $12.5 \mathrm{~cm}$ (left) and $18 \mathrm{~cm}$ (right) in step length after treadmill training with visual cues. ${ }^{15}$ Therefore, a mean stride length difference of $15 \mathrm{~cm}$ in Group 1 and $5 \mathrm{~cm}$ in Group 2 with a standard deviation of $8 \mathrm{~cm}$ was assumed for apriori sample size calculation. The required sample size was 11 participants in each group (power = $80 \%, \alpha$-level $=5 \%$ ).

Demographic and clinical features as well as gait characteristics were descriptively summarized. We used an exploratory testing procedure (without $\alpha$-adjustment) to detect which mobility parameters respond to the combined treatment strategy and to generate hypotheses for further investigation. Owing to the non-normally distributed continuous data, non-parametric tests were used. The MannWhitney $U$-test was performed to detect betweengroup differences and the Wilcoxon signed ranks analysis was carried out to compare within-group effects. The $\alpha$-level was set at $P<0.05$.

Data were analyzed using the SPSS statistical software package 20 (IL, Chicago).

\section{Results}

A total of 23 patients were allocated to the two training groups, 20 patients completed the training 


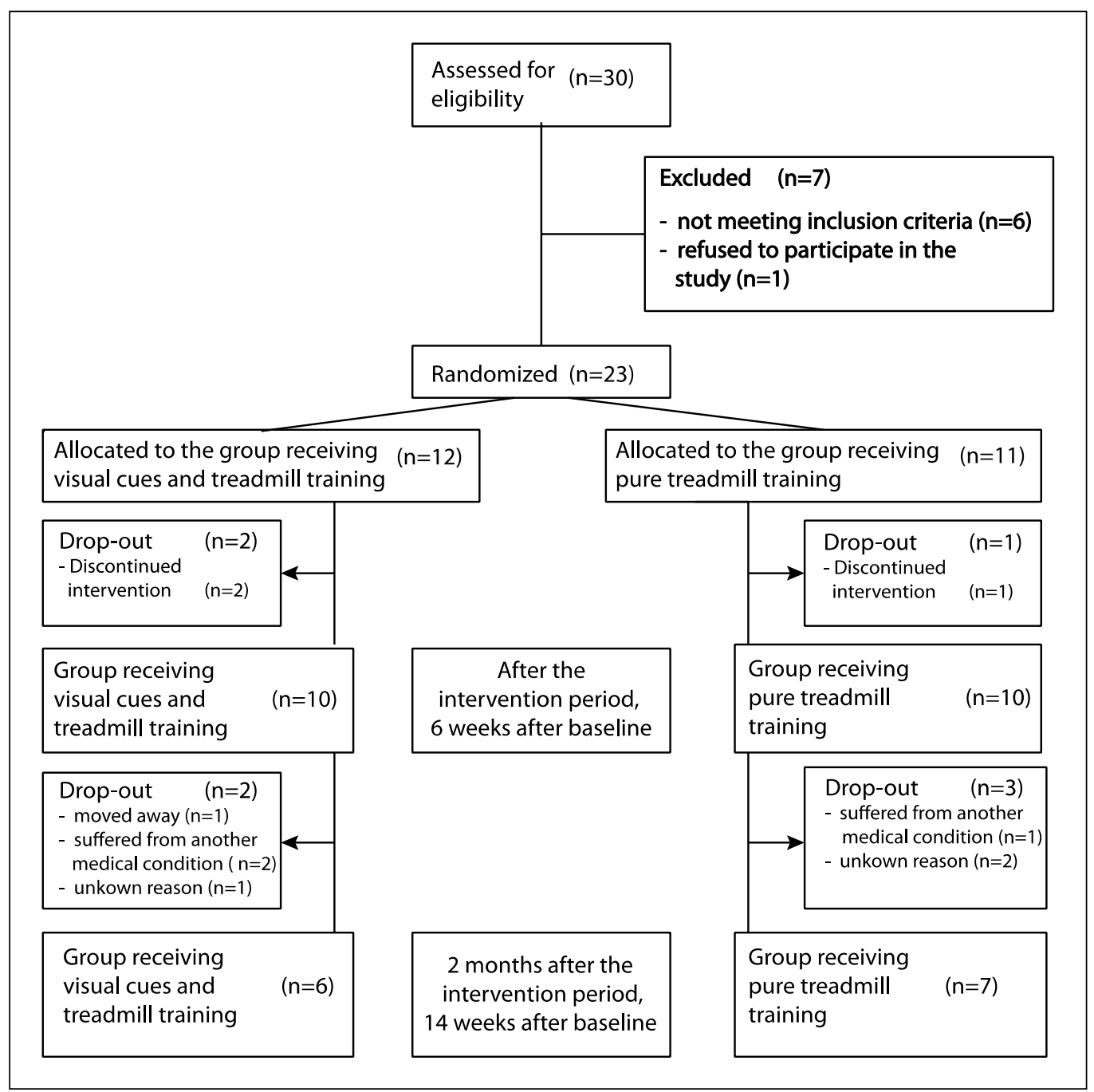

Figure 2. Flow diagram.

and 13 patients were assessed two months after the training period (Figure 2). Demographic and clinical characteristics of the patients are presented in Table 1. At baseline, there was no difference between the two groups in any of the demographic, clinical or outcome parameters.

Table 2 gives a summary of the outcome measures at each time of assessment. Change scores over time and differences between the two groups are presented in Table 3.

Within both groups, gait speed and stride length improved after the training period (Group 1: $p=0.000$ and $p=0.001$, respectively; Group 2: $p=0.001$ and $p=0.002$, respectively), whereas the time to complete the Timed Up and Go Test and the score of the UPDRS III decreased only in Group 1 ( $p=0.006$ and $p=0.019$, respectively). Cadence did not change within both groups $(p=0.665$ and $p=0.650$, respectively), indicating that the enhanced gait speed was achieved by increasing the stride length rather than the cadence. Upon follow-up after two months, both Group 1 and Group 2 showed lower values in gait speed $(p=0.046$ and $p=0.018$, respectively) and stride length $(p=0.028$ 
Table I. Demographic and clinical characteristics at baseline.

\begin{tabular}{lcc}
\hline & Group Ia & Group 2 $2^{\mathrm{b}}$ \\
\cline { 2 - 3 } & $n=10$ & $n=10$ \\
\hline Age, years Mean (SD) & $71.2(10.9)$ & $68.9(6.8)$ \\
Female gender $n(\%)$ & $8(80.0)$ & $6(60.0)$ \\
Disease duration, years Mean (SD) & $10.4(5.2)$ & $9.1(3.1)$ \\
Hoehn and Yahr stage Mean (SD) & $2.8(0.9)$ & $2.7(0.7)$ \\
MMSE Mean (SD) & $28.6(1.6)$ & $28.4(1.6)$ \\
\hline
\end{tabular}

UPDRS: Unified Parkinson's Disease Rating Scale (motor section); FoG-Q: Freezing of Gait-Questionnaire; MMSE: Mini-Mental State Examination.

aPatients receiving treadmill training in combination with visual cues.

bPatients receiving pure treadmill training.

Table 2. Outcome measures at baseline, after the training period and at two months follow-up.

\begin{tabular}{|c|c|c|c|c|c|c|c|}
\hline & & \multicolumn{3}{|c|}{ Group Ia } & \multicolumn{3}{|c|}{ Group $2^{b}$} \\
\hline & & $n$ & Mean (SD) & Median (range) & $n$ & Mean (SD) & Median (range) \\
\hline \multirow[t]{3}{*}{ Gait speed, km/h } & TI & 10 & $\mathrm{I} .7(0.8)$ & I.7 (I.3, 2.0) & 10 & $2.4(0.7)$ & $2.6(1.7,2.8)$ \\
\hline & $\mathrm{T} 2$ & 10 & $2.6(0.7)$ & $2.8(2.2,3.1)$ & 10 & $3.5(0.9)$ & $3.2(2.8,4.5)$ \\
\hline & $\mathrm{T} 3$ & 6 & $2.6(0.5)$ & $2.6(2.2,2.9)$ & 7 & $2.5(1 . I)$ & $2.1(1.5,3.9)$ \\
\hline \multirow[t]{3}{*}{ Stride length, $\mathrm{cm}$} & TI & 10 & $61.1(29.6)$ & $65.1(44.6,86.3)$ & 10 & $75.1(18.2)$ & $76.8(64.6,86.9)$ \\
\hline & $\mathrm{T} 2$ & 10 & $90.4(21.7)$ & $97.0(83.2,101.8)$ & 10 & $104.5(21.7)$ & $100.1(92.5,120.5)$ \\
\hline & T3 & 6 & $78.4(23.4)$ & $89.7(58.8,94.9)$ & 7 & $82.2(25.2)$ & $73.5(60.5,106.9)$ \\
\hline \multirow[t]{3}{*}{ Cadence, steps/min } & TI & 10 & $99.9(28.1)$ & $98.6(72.9,114.5)$ & 10 & $107.4(21.8)$ & $108.7(97.8,121.5)$ \\
\hline & $\mathrm{T} 2$ & 10 & $95.4(10.9)$ & $99.7(88.0,102.4)$ & 10 & $110.0(13.2)$ & II $2.4(102.9,119.0)$ \\
\hline & T3 & 6 & $95.6(8.4)$ & $99.0(86.1,102.6)$ & 7 & $99.7(23.1)$ & $102.3(75.2,119.4)$ \\
\hline \multirow[t]{3}{*}{ Timed Up and Go Test, s } & TI & 10 & I4.4 (6.8) & $12.5(7.5,18.1)$ & 10 & $10.9(4.7)$ & $10.2(7.1,13.9)$ \\
\hline & $\mathrm{T} 2$ & 10 & I I.8 (5.5) & $9.9(6.9,13.9)$ & 10 & $10.8(4.0)$ & $10.4(7.1,14.0)$ \\
\hline & T3 & 6 & $10.9(4.4)$ & $10.7(6.4,15.8)$ & 7 & $10.4(5.0)$ & $7.1(6.8,14.5)$ \\
\hline \multirow[t]{3}{*}{ UPDRS III } & TI & 10 & $28.9(13.8)$ & $26.0(14.8,43.3)$ & 10 & $25.3(15.1)$ & $25.5(9.8,36.8)$ \\
\hline & $\mathrm{T} 2$ & 10 & $23.8(13.5)$ & $24.5(12.5,33.8)$ & 10 & $23.4(10.1)$ & $26.0(12.8,31.5)$ \\
\hline & T3 & 6 & $21.8(13.4)$ & $18.5(10.5,38.0)$ & 7 & $28.2(13.7)$ & $23.5(19.5,35.5)$ \\
\hline \multirow[t]{3}{*}{ FOG-Q } & TI & 10 & $9.6(5.7)$ & $10.5(5.5,15.3)$ & 10 & $10.5(6.2)$ & II.5 (5.0, 16.3) \\
\hline & $\mathrm{T} 2$ & 10 & $10.0(6.9)$ & $10.0(3.8,15.5)$ & 10 & $9.8(6.5)$ & $10.5(3.0,16.0)$ \\
\hline & T3 & 6 & $3.2(4.1)$ & $2.5(0.0,5.0)$ & 7 & $4.2(4.5)$ & $3.0(2.3,5.5)$ \\
\hline
\end{tabular}

TI: baseline assessment; T2: assessment after the training period (six weeks after baseline); T3: assessment at two months follow-up ( 14 weeks after baseline); UPDRS: Unified Parkinson's Disease Rating Scale (motor section); FoG-Q: Freezing of GaitQuestionnaire.

aPatients receiving treadmill training in combination with visual cues.

bPatients receiving pure treadmill training.

and $p=0.008$, respectively) but the decrease was larger in Group 2 (Table 3). The time to complete the Timed Up and Go Test changed in neither Group $1(p=0.912)$ nor Group $2(p=0.237)$.
The increase of stride length in Group 1 was positively correlated with the score of the freezing of gait questionnaire $\left(\mathrm{r}_{\text {Spearman }}=0.87 ; p=0.001\right)$, suggesting that patients with higher freezing scores 
Table 3. Change in outcome measures after the training period and at two months follow-up.

\begin{tabular}{|c|c|c|c|c|c|c|c|c|}
\hline & & \multicolumn{3}{|c|}{ Group Ia } & \multicolumn{3}{|c|}{ Group $2^{b}$} & \multirow[t]{2}{*}{$p$-value } \\
\hline & & $n$ & $\begin{array}{l}\text { Change } \\
\text { Mean (SD) }\end{array}$ & Median (range) & $n$ & $\begin{array}{l}\text { Change } \\
\text { Mean (SD) }\end{array}$ & Median (range) & \\
\hline \multirow{2}{*}{$\begin{array}{l}\text { Gait speed, } \\
\mathrm{km} / \mathrm{h}\end{array}$} & $\mathrm{T} 2-\mathrm{TI}$ & 10 & $0.9(0.4)$ & $1.0(0.5,1.2)$ & 10 & I.I (0.7) & I.I $(0.5,1.7)$ & 0.579 \\
\hline & $\mathrm{T} 3-\mathrm{T} 2$ & 6 & $-0.4(0.3)$ & $-0.3(-0.7,-0.2)$ & 7 & $-0.9(0.5)$ & $-0.9(-1.1,-0.5)$ & $0.035^{c}$ \\
\hline \multirow{2}{*}{$\begin{array}{l}\text { Stride length, } \\
\mathrm{cm}\end{array}$} & $\mathrm{T} 2-\mathrm{TI}$ & 10 & $29.3(19.7)$ & $23.4(15.6,39.8)$ & 10 & $29.3(21.1)$ & $23.7(10.3,47.5)$ & 0.912 \\
\hline & $\mathrm{T} 3-\mathrm{T} 2$ & 6 & $-7.3(10.4)$ & $-7.8(-17.5,3.2)$ & 7 & $-23.6(15.3)$ & $-24.4(-27.8,-12.5)$ & $0.038^{c}$ \\
\hline \multirow{2}{*}{$\begin{array}{l}\text { Cadence, } \\
\text { steps } / \mathrm{min}\end{array}$} & $\mathrm{T} 2-\mathrm{TI}$ & 10 & $-4.5(31.8)$ & $3.2(-12.1,11.9)$ & 10 & $2.7(18.1)$ & $4.8(-7.9,13.1)$ & 0.684 \\
\hline & $\mathrm{T} 3-\mathrm{T} 2$ & 6 & $-3.4(7.2)$ & $-2.5(-9.1,1.9)$ & 7 & $-6.1(15.3)$ & $-7.3(-16.3,-1.8)$ & 0.366 \\
\hline \multirow{2}{*}{$\begin{array}{l}\text { Timed Up and } \\
\text { Go Test, s }\end{array}$} & $\mathrm{T} 2-\mathrm{TI}$ & 10 & $-3.6(2.3)$ & $-2.3(-4.2,-0.6)$ & 10 & $-0.2(1.6)$ & $-0.1(-1.5,1.5)$ & $0.023^{c}$ \\
\hline & $\mathrm{T} 3-\mathrm{T} 2$ & 6 & $-0.1(1.6)$ & $-0.2(-0.9,1.3)$ & 7 & $-0.5(2.6)$ & $-0.7(-1.9,-0.2)$ & 0.445 \\
\hline \multirow[t]{2}{*}{ UPDRS III } & $\mathrm{T} 2-\mathrm{TI}$ & 10 & $-5.1(5.0)$ & $-6.0(-9.5,-1.0)$ & 10 & $-1.9(8.7)$ & $-1.0(-7.0,4.0)$ & 0.218 \\
\hline & T3-T2 & 6 & $3.3(5.6)$ & $3.0(-0.3,6.2)$ & 6 & $4.8(10.5)$ & $2.5(-1.8, \mid 1.3)$ & 0.937 \\
\hline \multirow[t]{2}{*}{ FOG-Q } & $\mathrm{T} 2-\mathrm{TI}$ & 10 & $0.4(3.3)$ & $0.0(-1.3,2.5)$ & 10 & $-0.7(1.8)$ & $-1.5(-2.0,0.5)$ & 0.218 \\
\hline & T3-T2 & 6 & $-4.0(3.5)$ & $-4.5(-6.8,0.0)$ & 6 & $-8.3(5.8)$ & $-10.0(-13.3,-2.3)$ & 0.240 \\
\hline
\end{tabular}

TI: baseline assessment; T2: assessment after the training period (six weeks after baseline); T3: assessment at two months follow-up ( 4 weeks after baseline); UPDRS: Unified Parkinson's Disease Rating Scale (motor section); FoG-Q: Freezing of GaitQuestionnaire.

apatients receiving treadmill training in combination with visual cues.

bPatients receiving pure treadmill training.

'Significant difference between the two groups $(p<0.05)$

showed a greater benefit from the combined training strategy than patients with lower freezing scores. No such correlation was found for Group 2 $\left(\mathrm{r}_{\text {Spearman }}=0.23 ; p=0.521\right)$.

\section{Discussion}

The findings of this study support the hypothesis that visual cueing with treadmill training has more beneficial impact on gait performance than pure treadmill training. First, only the combined training strategy helped to improve functional walking performance, assessed by the Timed Up and Go Test. Second, gait speed and stride length increased in both training groups, but patients who received the additional cues sustained better results over two months.

In addition, visual cues during treadmill training seemed to be particularly successful in enhancing stride length of patients suffering from freezing of gait.

Some major limitations of this study need to be considered. Although not statistically significant, Group 2 scored better in several baseline assessments, particularly the Timed Up and Go Test. This means that there was less potential for improvement and a possible ceiling effect of performance in Group 2. The following aspects might have caused adverse effects on the validity of the study. Like in most pilot clinical trials, the sample size was small and cannot be representative for all patients with Parkinson's disease. Furthermore, 35\% of the patients were lost to follow-up, reducing the number of patients to six and seven in the two groups. Therefore, results may be subject to type II errors as well as attrition bias and need to be interpreted with caution. Blinding of participants and the physiotherapist who supervised the training sessions was not possible owing to the nature of the interventions. The fact that participants were aware of the group allocation may have caused a decline in motivation, and thus, a potential lack of treatment success, especially among the patients receiving only treadmill training without the additional cues. To improve the participant's compliance, they were offered to receive the respective other treatment after cessation of the study. Experimenter bias could have been introduced by the physiotherapist during the training sessions. To counter this, the therapist was instructed 
to avoid any kind of encouragement. As the assessors were not blinded, observer bias may have affected the results. This weakness could have particularly influenced the walking speed on the treadmill. However, patients were instructed to adjust their preferred and maximum speeds individually while only being supervised by the assessor, and furthermore, mobility measures were objective. It is worthwhile to initiate a multi-center assessorblinded randomized controlled trial to evaluate the efficacy of visual cues during treadmill training within a large sample of patients with Parkinson's disease. The Timed Up and Go Test would be an appropriate primary outcome measure.

It is well known that patients with Parkinson's disease compensate for short stride length by increasing the cadence. ${ }^{1}$ In this study, both training groups showed improvement in gait speed and stride length while cadence did not change. This may indicate that patients who train on a treadmill learn how to modulate gait speed by regulating stride length rather than cadence. Previous research already found that treadmill walking facilitates positive effects on the spatiotemporal gait parameters, probably owing to the repetitive and pacedictating stimulation of the walking task..$^{10,11,12}$

Frazzitta et al. studied the efficacy of treadmill training with visual cues presented on a screen compared with conventional therapy with cues in Parkinson patients with freezing. ${ }^{21}$ Similar to our results, they found that the combination was more effective to improve gait performance. Most of all, patients scored better in the 6-minute walking test. Luessi and colleagues investigated the interaction of treadmill walking and visual cues attached to the belt on the spatiotemporal gait parameters. ${ }^{22}$ Under the cued condition, stride length increased while cadence decreased. Furthermore, they found out that the effect of the visual cues was related to gait speed, meaning that the positive influence on stride length and gait speed was greater at slow velocities (around $1-2 \mathrm{~km} / \mathrm{h}$ ). Together with our data, this study suggests that the combined intervention is most efficient at slow training speeds.

Better results in the Timed Up and Go Test could only be achieved in patients who underwent the combined training, and these patients seemed to sustain the gain in functional gait performance over two months. This finding proposes that the visual stimulation helps to transfer and integrate the training effects into walking on the natural ground. The patients in Group 1 were interviewed after the training period to find a possible explanation. The severely affected patients reported to mentally visualize their footprints on the ground in front of them whenever they experience difficulties generating continuous walking, whereas the mildly affected patients reported improvements without using any specific strategy. These observations may demonstrate that severely affected patients are dependent on visual information (even if it is imaginary) to focus their attention on walking, while less affected patients still have the ability to improve walking performance by motor learning. This hypothesis is supported by the findings of Morris and colleagues. ${ }^{23}$ They recommend compensatory techniques, particularly visual and auditory cues, for the moderate to severe stage and motor skill learning strategies, namely repetitive task-complex training, for the early stage of the Parkinson's disease. ${ }^{23}$

Freezing of gait was positively correlated with the increase in stride length after receiving visual cues in combination with treadmill training. Freezing is the clinical symptom of impaired automatic motor control caused by a functional miscommunication of the locomotor network, manifested as reduced connectivity between the supplementary motor area and the subthalamic nucleus, and greater connectivity between the supplementary motor area and the mesencephalic and cerebellar motor regions. ${ }^{24} \mathrm{~A}$ recent review presents four theoretical models that might explain the episodic emergence of freezing. ${ }^{25}$ Two of them, the threshold and the interference model, ${ }^{25,26,27}$ best describe the superior effect mechanism of the combined training in freezers. The threshold model assumes that freezing is induced by the accumulation of motor impairments, such as reduced step scaling, coordination and symmetry, and decreased gait rhythmicity, resulting in a breakdown of locomotion. ${ }^{25,26}$ Treadmill walking could help to generate a more rhythmic and regular gait pattern, thereby preventing the threshold from reaching the 
level of freezing. The interference model proposes that freezing occurs owing to the incapacity to process concurrent cognitive, limbic and motor input, which again leads to a breakdown of locomotion. ${ }^{25,27}$ Visual cues may help to correct the interference between neural circuits by channeling information through intact pathways. ${ }^{27,28}$ As the pharmacological treatment of freezing remains difficult, ${ }^{28}$ the combination of treadmill training and visual cueing could guide the way to designing successful therapy approaches for these patients.

\section{Clinical messages}

- Adding visual cues during treadmill training improves the gait performance in patients with a moderate to severe stage of Parkinson's disease, and particularly in patients with freezing.

- The combined training helps to translate the gait improvements from treadmill walking into normal walking on the natural surface.

\section{Acknowledgements}

The authors would like to thank the patients for participating in the study.

In memoriam: Dr Josef Ilmberger. In December 2014, Dr Josef Ilmberger unexpectedly died leaving his colleagues saddened by the loss of a great scientist and warm-hearted friend. We wish to express our deepest appreciation for his valuable work in the fields of rehabilitation and neuropsychology.

\section{Conflict of interest}

The authors declare that there is no conflict of interest.

\section{Contributors}

CS designed and coordinated the study, analyzed the data and drafted the manuscript. AE was substantially involved in the study design and data collection. $\mathrm{KB}$, AP and OP made contributions to the conception of the study and the interpretation of the data. JI initiated the study, monitored its progress and helped to analyze the data. All authors read and approved the final manuscript.

\section{Funding}

This work was supported by the German Federal Ministry of Economics and Technology - Zentrales Innovationsprogramm Mittelstand [Grant number: KF2249501FO9].

\section{References}

1. Morris ME, Iansek R, Matyas TA and Summers JJ. The pathogenesis of gait hypokinesia in Parkinson's disease. Brain 1994; 117: 1169-1181.

2. Boonstra TA, van der Kooij H, Munneke $M$ and Bloem BR. Gait disorders and balance disturbances in Parkinson's disease: Clinical update and pathophysiology. Curr Opin Neurol 2008; 21: 461-471.

3. Rahman S, Griffin HJ, Quinn NP and Jahanshahi M. Quality of life in Parkinson's disease: The relative importance of the symptoms. Mov Disord 2008; 23: 1428-1434.

4. Soh SE, McGinley JL, Watts JJ, Iansek R and Morris ME. Health-related quality of life of Australians with Parkinson disease: A comparison with international studies. Physiother Can 2012; 64: 338-346.

5. Spaulding SJ, Barber B, Colby M, Cormack B, Mick T and Jenkins ME. Cueing and gait improvement among people with Parkinson's disease: A meta-analysis. Arch Phys Med Rehabil 2013; 94: 562-570.

6. Morris ME, Iansek R, Matyas TA and Summers JJ. Stride length regulation in Parkinson's disease. Normalization strategies and underlying mechanisms. Brain 1996; 119: $551-568$

7. Rochester L, Baker K, Hetherington V, et al. Evidence for motor learning in Parkinson's disease: Acquisition, automaticity and retention of cued gait performance after training with external rhythmical cues. Brain Res 2010; 1319: 103-111.

8. Rubinstein TC, Giladi N and Hausdorff JM. The power of cueing to circumvent dopamine deficits: A review of physical therapy treatment of gait disturbances in Parkinson's disease. Mov Disord 2002; 17: 1148-1160.

9. Griffin HJ, Greenlaw R, Limousin P, Bhatia K, Quinn NP and Jahanshahi $\mathrm{M}$. The effect of real and virtual visual cues on walking in Parkinson's disease. J Neurol 2011; 258: 991-1000.

10. Frenkel-Toledo S, Giladi N, Peretz C, Herman T, Gruendlinger L and Hausdorff JM. Treadmill walking as an external pacemaker to improve gait rhythm and stability in Parkinson's disease. Mov Disord 2005; 20: 11091114.

11. Bello O, Marquez G, Camblor M and Fernandez-DelOlmo M. Mechanisms involved in treadmill walking improvements in Parkinson's disease. Gait Posture 2010; 23: $118-123$.

12. Herman T, Giladi N and Hausdorff JM. Treadmill training for the treatment of gait disturbances in people with Parkinson's disease: A mini-review. J Neural Transm 2009; 116: 307-318. 
13. Mehrholz J, Friis R, Kugler J, Twork S, Storch A and Pohl M. Treadmill training for patients with Parkinson's disease. Cochrane Database Syst Rev 2010; 20: 1-33.

14. Miyai I, Fijimoto Y, Yamamoto H, et al. Long-term effect of body weight-supported treadmill training in Parkinson's disease: A randomized controlled trial. Arch Phys Med Rehabil 2002; 83: 1370-1373.

15. Schlick C, Struppler A, Boetzel K, Plate A and Ilmberger J. Dynamic visual cueing in combination with treadmill training for gait rehabilitation in Parkinson disease. Am J Phys Med Rehabil 2012; 91: 75-79.

16. Goetz CG, Poewe W, Rascol O, et al. Movement Disorder Society Task Force report in the Hoehn and Yahr staging scale: Status and recommendations. Mov Disord 2004; 19: 1020-1028.

17. Lopez MN, Charter RA, Mostafavi B, Nibut LP and Smith WE. Psychometric properties of the Folstein Mini-Mental State Examination. Assessment 2005; 12: 137-144.

18. Morris S, Morris ME and Iansek R. Reliability of measurements obtained with the "Timed up \& go test" in people with Parkinson disease. Phys Ther 2001; 81: 810-818.

19. Vogler A, Janssens J, Nyffeler T, Bohlhalter S and Vanbellingen T. German translation and validation of the "freezing of gait questionnaire" in patients with Parkinson's disease. Parkinsons Dis 2015; Article ID 982058.

20. Movement Disorder Society Task Force on Rating Scales for Parkinson's Disease. The Unified Parkinson's Disease Rating Scale (UPDRS): Status and recommendations. Mov Disord 2003; 18: 738-750.
21. Frazzitta G, Maestri R, Uccellini D, Bertotti G and Abelli P. Rehabilitation treatment of gait in patients with Parkinson's disease with freezing: A comparison between two physical therapy protocols using visual and auditory cues with or without treadmill training. Mov Disord 2009; 24: 1139-1143.

22. Luessi F, Mueller LK, Breimhorst M and Vogt T. Influence of visual cues on gait in Parkinson's disease during treadmill walking at multiple velocities. J Neurol Sci 2012; 314: 78-82.

23. Morris ME, Martin CL and Schenkman ML. Striding out with Parkinson disease: Evidence-based physical therapy for gait disorders. Phys Ther 2010; 90: 280-288.

24. Fling BW, Cohen RG, Mancini M, et al. Functional reorganization of the locomotor network in Parkinson patients with freezing of gait. Plos One 2014; 9: e100291.

25. Nieuwbower A and Giladi N. Characterizing freezing of gait in Parkinson's disease: Models of an episodic phenomenon. Mov Disord 2013; 28: 1509-1519.

26. Plotnik M, Giladi N and Hausdorff JM. Is freezing of gait in Parkinson's disease a result of multiple gait impairments? Implications for treatment. Parkinsons Dis 2012; 2012: 459321.

27. Lewis SJG and Barker RA. A pathophysiological model of freezing of gait in Parkinson's disease. Parkinsonism Relat Disord 2009; 15: 333-338.

28. Nutt JG, Bloem BR, Giladi N, Hallett M, Horak FB and Nieuwboer A. Freezing of gait: Moving forward on a mysterious clinical phenomenon. Lancet Neurol 2011; 10: 734-744. 